\title{
AN OBSTRUCTION TO FINITENESS OF CW-COMPLEXES
}

\author{
BY C. T. C. WALL
}

\author{
Communicated by W. S. Massey, November 4, 1963
}

A cell structure is a convenient means of describing a space; thus it is important to reduce such a structure to a simpler one when possible. For example, it remains unsolved whether a compact topological manifold (or more generally, ANR) has the homotopy type of a finite CW-complex. According to Milnor [2], this would follow from the conjecture that any CW-complex which is dominated by a finite complex has the homotopy type of a finite complex, but we show below that this is false.

Let $X$ be a connected CW-complex, with universal cover $\tilde{X}$, and fundamental group $\pi$ with (integral) group ring $\Lambda$. Consider the following conditions:

(i) $X$ is dominated by a complex of finite type (i.e., one with a finite number of cells of each dimension),

(ii) $\pi$ and all $H_{i}(\tilde{X})$ are countable,

(iii) $)_{N}$ For $N<i, H_{i}(\tilde{X})=0$ and $H^{i}(X ; ß)=0$ for all coefficient bundles $B$ (in the sense of Steenrod; generalised to non-abelian coefficients if $i=2$ ).

Our results are as follows:

(A) If (i) holds, $X$ is homotopy equivalent to a complex of finite type.

(B) If $\Lambda$ is noetherian, (i) is equivalent to: $\pi$ is finitely presented, and all $H_{i}(\tilde{X})$ are finitely generated $\Lambda$-modules.

(C) If $X$ is dominated by a countable complex, it is homotopy equivalent to one; this condition is equivalent to (ii).

(E) If (iii) $)_{N}$ holds, and $N \neq 2, X$ has the homotopy type of an $N$ dimensional complex, countable if (ii) holds.

(F) $X$ is dominated by a finite complex if and only if (i) and some $(\text { iii) })_{N}$ hold. When this is the case, and $N \geqq 2$, there is an obstruction $\theta(X)$ in the projective class group $\tilde{K}^{0}(\Lambda)$, which depends only on the homotopy type of $X$, and is zero for $X$ finite. If $\theta(X)=0, X$ has the homotopy type of a finite complex of dimension $\max (3, N)$. For $N \geqq 2$, any finite complex $K$ of dimension $N$, and $\alpha \in \widetilde{K}^{0}\left(\pi_{1}(K)\right.$ ), there is a complex $X$, with the $(N-1)$-type of $K$, satisfying (i) and $(\text { iii })_{N}$, and with $\theta(X)=\alpha$.

The proofs are mostly by induction; we obtain complexes $K^{r}$ and $r$-connected maps $\phi: K \rightarrow X$, where $K$ is finite in (A), countable in (C). We then prove that $\pi_{r+1}(\phi)$ is finitely generated (over $\Lambda$ ) in (A), 
and countable in (C), and that we can always use a set of $\Lambda$-generators $(r \geqq 2)$ of $\pi_{r+1}(\phi)$ to attach $(r+1)$-cells to $K$, and extend $\phi$ over them, to obtain an $(r+1)$-connected map. If $X$ satisfies (iii) ${ }_{N}$, and $r=N-1$, then $\pi_{N}(\phi)$ is a projective $\Lambda$-module; when it is free, the process above gives a homotopy equivalence.

The crucial step in the proof of (A), which is used again in $(F)$ in showing that $\theta(X)$ is well defined, is the following lemma of Whitehead [5]:

Let $P$ be a finite connected complex, $K$ a connected subcomplex with $\pi_{r}(P, K)=0$ for $1 \leqq r<n$. Then there is a formal deformation (and so homotopy equivalence) $D: P \rightarrow Q$ rel $K$ such that for $r<n$, $Q$ has no $r$-cells outside $K$, and for $r \geqq n+2, Q$ has the same number of $r$-cells outside $K$ as $P$ does.

We observe that there is an interesting analogy between our obstruction in $\tilde{K}^{0}(\Lambda)$ (which is the Grothendieck group of finitely generated projective modulo free modules) to existence of finite complexes equivalent to $X$, and Whitehead's obstruction in $K^{1}(\Lambda)$ (reduced by $\pm \pi)$ to their uniqueness up to formal deformation [5]. We refer the reader to Bass and Schanuel [1] for the relation between $K^{0}(\Lambda)$ and $K^{1}(\Lambda)$.

According to Swan [4], $\tilde{K}^{0}(\Lambda)$ is finite, if $\pi$ is, and by $\operatorname{Rim}$ [3], if $\pi$ is cyclic of prime order, $\tilde{K}^{0}(\Lambda)$ is isomorphic to the ideal class group of the corresponding cyclotomic field. This gives several examples both of zero and of nonzero $\widetilde{K}^{0}(\Lambda)$.

The main unsatisfactory feature of the above is our inability to construct 2-dimensional complexes under appropriate hypotheses. Roughly speaking, by the time we have enough 2-cells to give relations between the generators of the fundamental group, we may have too many for the homology.

\section{REFERENCES}

1. H. Bass and S. Schanuel, The homotopy theory of projective modules, Bull. Amer. Math. Soc. 68 (1962), 425-428.

2. J. W. Milnor, On spaces having the homotopy type of a CW-complex, Trans. Amer. Math. Soc. 90 (1959), 272-280.

3. D. S. Rim, Modules over finite groups, Ann. of Math. (2) 69 (1959), 700-712.

4. R. G. Swan, Induced representations and projective modules, Ann. of Math. (2) 71 (1960), 552-578.

5. J. H. C. Whitehead, Simple homotopy types, Amer. J. Math. 72 (1950), 1-57.

Trinity College, Cambridge, England 